\title{
Event-triggered Prescribed Settling Time Consensus Control of Uncertain Nonlinear Multiagent Systems with Transient Performance
}

\section{Zicong Chen}

Guangdong University of Technology

Jianhui Wang ( $\nabla 7517619 @ q q . c o m$ )

Guangzhou University https://orcid.org/0000-0003-4766-6216

\section{Li Zhang}

Guangzhou Real Estate and Land Management Vocational

\section{Kemao Ma}

Harbin Institute of Technology

Yanhui Liu

Guangzhou University

\section{Research Article}

Keywords: Consensus Control , Transient Performance, Prescribed Settling Time, Event-triggered control

Posted Date: April 8th, 2021

DOI: https://doi.org/10.21203/rs.3.rs-397534/v1

License: (c) (1) This work is licensed under a Creative Commons Attribution 4.0 International License. Read Full License 


\title{
Event-triggered Prescribed Settling Time Consensus Control of Uncertain Nonlinear Multiagent Systems with Transient Performance
}

\author{
Zicong Chen · Jianhui Wang · Li Zhang - Kemao Ma · Yanhui Liu
}

Received: date / Accepted: date

\begin{abstract}
Multiagent systems are often applied in unmanned aerial vehicle formations, multi-manipulator coordinated, traffic vehicle control and other fields, which have attracted a lot of attention from scholars. In this paper, considering the given transient performance of multiagent systems, its synchronization error converges to the predefined interval by designing the performance function. In the meanwhile, through the designed nonlinear transformation of synchronization error, a prescribed setting time tracking control is realized, which can ensure that the multiagent systems are uniformly bounded convergent. In addition, event-triggered strategy is introduced to economize the utilization of multiagent systems' communication resources. Finally, theoretical analysis and simulation research demonstrates the effectiveness of the proposed method.
\end{abstract}

\section{Z.C. Chen}

the school of Automation, Guangdong University of Technology, Guangzhou 510006, China

E-mail: chenzicong_ch@163.com

J.H. Wang (四)

the School of Mechanical and Electric Engineering, Guangzhou University, Guangzhou 510006, China

E-mail: jhwang@gzhu.edu.cn

L. Zhang

the School of Guangzhou Real Estate and Land Management

Vocational, Guangzhou, 510320, China

E-mail: zhangli_0296@163.com

K.M. Ma

the school of Harbin Institute of Technology, Harbin 150000, China

E-mail: makemao@hit.edu.cn

Y.H. Liu

State Key Laboratory for Seismic Reduction/Control and Structural Safety (Cultivation), Guangzhou University,

Guangzhou 510006, China

E-mail: Liuyanhui2012@163.com
Keywords Consensus Control · Transient Performance · Prescribed Settling Time · Event-triggered control

\section{Introduction}

Consensus control has received extensive attention from both theoretical and practical perspectives $[1,2]$. The value of synchronization error is a key indicator to measure the performance of multiagent systems (MASs). Most of the existing methods can ensure that the synchronization error eventually converges to a small residual set. But MASs' transient performance is equally important, which can avoid related interference between agents effectively [3-13]. In [3], by properly designing certain performance boundaries, connection maintenance and conflict avoidance between adjacent agents were realized. In $[5,6]$, in order to obtain better system transient performance, a method combining barrier Lyapunov function and exponential function was developed. With the aid of dynamic surface control technique, the scholars in [10] established distributed consensus controllers to ensure the transient performance of MASs. In [13], the issue of consensus reaching with prescribed transient behavior for a group of double-integrator agents was investigated. The above methods can guarantee the transient performance of the system well. Further, in practical engineering, whether the MASs can guarantee its transient performance within finite-time is of great significance.

System stability is the foundation of all expected performance. In system analysis, Lyapunov stability theory can only help us get asymptotically stable results of the system. It implies that as time goes to infinity, system state converges to the equilibrium point. With this 
in mind, finite-time control comes into being [14-16] and related results continues to emerge [17-26]. In [17], under the condition of being disturbed, agents' state can be guaranteed to achieve synchronization in finitetime. In [18], the tracking error of the considered system remains in a small neighborhood of the origin after a finite period of time. In [20], the finite-time stability theory was applied to the control design to deal with the stabilization time problem. In [24], the finite-time output tracking issue for nonlinear systems with multiple mismatched disturbances was investigated. The above literatures prove that they are able to achieve finite-time stable from perspective of theorems. But the specific stabilization time is unknown. In [25], with the aid of the distributed observer and the designed power integrator, a novel nonlinear protocol is established to track the the estimated leader state in a prescribed finite-time. In [26], a new prescribed-time distributed control method for consensus and containment of networked multiple systems was addressed. These methods can achieve the system convergence within the preset time. However, how to ensure the MASs with given transient performance uniformly bounded convergent within the prescribed settling time remains an open issue.

Moreover, MASs' signal transmission is frequent, which will occupy a large amount of MASs' communication resources. For this situation, event-triggered control provides a approach to handle this problem. Different from the previous method of time-triggered, the sampling and control updates of event-triggered strategy depend on the predefined conditions [27-37]. The scholars in [27] systematically introduced some results of distributed event-triggered mechanism in dynamical systems. In [30], based on two different threshold strategy, a switching threshold strategy was proposed. In [31], an one-bit signal transmission, which only need to transmit one-bit signal to update the control signal, was investigated to further save communication resources. In [34], periodic event-triggered was considered, where the event-checkings are only implemented at periodic time instants. Further, in order to avoid continuous monitoring of system status or input, self-triggering control has also been developed [35-37]. Obviously, event-triggered strategy could significantly bring down the sampling frequency and control execution while maintaining the specified control performance. However, the above methods did not consider the system's given transient performance. How to balance the given transient performance of MASs and the pressure of communication resources is a challenge. Therefore, it is worth further investigating on the event- triggered consensus control for MASs with given transient performance.

As the above discussion goes further, how to ensure that MASs with given transient performance uniformly bounded converges within prescribed settling time while balancing the tracking performance and communication resources of the system is extremely challenging. The motivation of this study is to develop an eventtriggered prescribed setting time consensus control for uncertain nonlinear MASs with transient performance, which have the following main contributions.

1) Through finite-time control technology, many results are obtained that system state converges to a uniformly bounded convergence region in finitetime. Note that reaching a stable state within the specified time is meaningful to practical engineering system. However, while improving the fast tracking performance of MASs, the transient performance would be weakened. In this paper, a nonlinear transformation of the synchronization error is implemented to guarantee MASs' transient performance. Further, a prescribed setting time tracking control is established to ensure that the MASs are uniformly bounded convergent. The prescribed setting time convergence performance and transient performance of MASs are balanced well.

2) The signal transmission of MASs is frequent, but practical system communication resources are restricted. When the considered system is required to have given transient performance, it would bring certain challenges to the consumption of control communication resources. In order to deal with the contradiction, this study proposes an adaptive neural event-triggered control method, which can economize the utilization of communication resources effectively.

The paper will be expanded as follows. The consensus control issue will be formulated in Section 2. The detailed design and analysis process of the proposed method will be given in Section 3. And numerical example and practical example are carried on in Section 4. In Section 5, the conclusion will be presented.

\section{Problem Formulation and Preliminaries}

\subsection{Model and Definitions}

First of all, a class of MASs is considered as follows.

$\left\{\begin{array}{l}\dot{x}_{i, l}=x_{i, l+1}+f_{i, l}\left(x_{i}\right), l=1,2, \ldots, n-1, \\ \dot{x}_{i, n}=u_{i}+f_{i, n}\left(x_{i}\right), \\ y_{i}=x_{i, 1}\end{array}\right.$ 
where $u_{i} \in R$ is system input, $y_{i} \in R$ is system output. $x_{i}=\left[x_{i, 1}, x_{i, 2}, \ldots, x_{i, n}\right]^{T} \in R^{n}$ denotes the $i t h$ agent's state vector, $i=1,2, \ldots, N . f_{i, l}\left(x_{i}\right)$ and $f_{i, n}\left(x_{i}\right)$ represent unknown smooth functions, which will be abbreviate to $f_{i, l}$ and $f_{i, n}$ in subsequent expressions for simplicity.

Assumption 1: The output of the leader of MASs is known, smooth and bounded. Besides, its derivative exists.

Lemma 1 [38]: For $\forall \beta \in R, \tau>0$, the relationship in (2) holds .

$0 \leqslant \beta-\beta \tanh \left(\frac{\beta}{\tau}\right) \leqslant 0.2785 \tau$

where $-\beta \tanh \left(\frac{\beta}{\tau}\right) \leqslant 0$.

Lemma 2 [39]: For $\forall(x, y) \in R^{2}$, the following relationship can be obtained.

$x y \leqslant \frac{\xi}{\alpha}|x|^{\alpha}+\frac{1}{\beta \xi^{\beta}}|y|^{\beta}$,

where $\alpha>1, \beta>1, \xi>0,(\alpha-1)(\beta-1)=1$.

\subsection{Graph Theory}

The communication topology between agents is described by $\mathcal{G}=(\mathcal{V}, \mathcal{E}) . \mathcal{V}=\left\{\mathcal{V}_{1}, \mathcal{V}_{2}, \ldots, \mathcal{V}_{N}\right\}$ and $\mathcal{E} \subseteq \mathcal{V} \times \mathcal{V}$ denote relevant vertices and edges. The adjacency matrix of $\mathcal{G}$ is $\mathcal{A}=\left[a_{i j}\right]_{N \times N}$. If $\left(\mathcal{V}_{j}, \mathcal{V}_{i}\right) \in \mathcal{E}$, the note $i$ could receive message from the node $j, a_{i j}>0$, and $a_{i j}=0$ otherwise. $\mathcal{N}_{i}=\left\{\mathcal{V}_{j} \mid\left(\mathcal{V}_{j}, \mathcal{V}_{i}\right) \in \mathcal{E}, i \neq j\right\}$ denotes the set of neighbors. Further, $\mathcal{L}=\mathcal{D}-\mathcal{A} \in R^{N \times N}$ is an Laplacian matrix with $\mathcal{D}=\operatorname{diag}\left(d_{1}, d_{2}, \ldots, d_{N}\right) \in R^{N \times N}$, $d_{i}=\sum_{j \in \mathcal{N}_{i}} a_{i j}$.

Definition 1: The definition of MASs' synchronization error is presented as

$E_{i}=b_{i}\left(y_{i}-y_{d}\right)+\sum_{j \in \mathcal{N}_{i}} a_{i j}\left(y_{i}-y_{j}\right)$

where $b_{i} \geqslant 0$ represents the weight of edge from the node $i$ to the leader. When the $i$ th follower could receive information from the leader, $b_{i}>0$. Otherwise, $b_{i}=0$. And there will be one follower can communicate with the leader at least.

Lemma 3 [40]: Define $\mathcal{B}=\operatorname{diag}\left\{b_{i}\right\} \in R^{N \times N}$ with $b_{i}>0$. Combined with the definition of the Laplacian matrix $\mathcal{L}$, it can be known that $\mathcal{L}+\mathcal{B}$ is nonsingular.

Lemma 4 [40]: Define that $E=\left[E_{1}, E_{2}, \ldots, E_{N}\right]^{T}, y=$ $\left[y_{1}, y_{2}, \ldots, y_{N}\right]^{T}, Y_{d}=\underbrace{\left[y_{d}, y_{d}, \ldots, y_{d}\right]}_{N}$, the tracking error $y-Y_{d}$ holds the following inequality:

$\left\|y-Y_{d}\right\| \leqslant \frac{\|E\|}{\vartheta}$ where $\vartheta$ is the minimum singular value of the matrix $\mathcal{L}+\mathcal{B}$.

\subsection{The Transformed System for Given Transient} Performance

In this study, in order to achieve the given transient performance of synchronization error $E_{i}$, choose the performance function as $\eta(t)=\left(\eta_{0}-\eta_{\infty}\right) e^{-\gamma t}+\eta_{\infty}$, which satisfies the condition (6). $\eta_{0}>0, \eta_{\infty}>0$ and $\gamma>0$ are design parameters, $\eta(0)=\eta_{0}, \lim _{t \rightarrow \infty} \eta(t)=\eta_{\infty}$, $\eta_{0}>\eta_{\infty}$.

$-\delta_{\min } \eta(t)<E_{i}<\delta_{\max } \eta(t)$,

where $t \geqslant 0, \delta_{\min }>0, \delta_{\max } \leqslant 1$.

Define a strictly increasing function $\rho\left(z_{i, 0}\right)$, which satisfies

$-\delta_{\min }<\rho\left(z_{i, 0}\right)<\delta_{\max }$,

where $\rho\left(z_{i, 0}\right)=\frac{\delta_{\max } e^{\left(z_{i, 0}+s\right)}-\delta_{\min } e^{-\left(z_{i, 0}+s\right)}}{e^{\left(z_{i, 0}+s\right)}+e^{-\left(z_{i, 0}+s\right)}}, s=\frac{1}{2} \ln \left(\frac{\delta_{\min }}{\delta_{\max }}\right)$.

By combining (6) and (7), one has

$E_{i}=\eta(t) \rho\left(z_{i, 0}\right)$.

Thus

$$
\begin{aligned}
z_{i, 0} & =\rho^{-1}\left(\frac{E_{i}}{\eta(t)}\right) \\
& =\frac{1}{2} \ln \left(\frac{\delta_{\max } \sigma(t)+\delta_{\max } \delta_{\min }}{\delta_{\max } \delta_{\min }-\delta_{\min } \sigma(t)}\right),
\end{aligned}
$$

where $\sigma(t)=\frac{E_{i}}{\eta(t)}$.

Take the time derivative of $z_{i, 0}$ yields

$$
\begin{aligned}
\dot{z}_{i, 0}= & \frac{\partial \rho^{-1}}{\partial \sigma}=\frac{1}{2}\left(\frac{1}{\sigma+\delta_{\min }}-\frac{1}{\sigma-\delta_{\max }}\right)\left(\frac{\dot{E}_{i}}{\eta}-\frac{E_{i} \dot{\eta}}{\eta^{2}}\right) \\
= & \xi\left(\left(\left(b_{i}+d_{i}\right)\left[f_{i, 1}\left(x_{i}\right)+x_{i, 2}\right]-b_{i} \dot{y}_{d}\right.\right. \\
& \left.\left.-\sum_{j \in \mathcal{N}_{i}} a_{i j}\left(f_{j, 1}\left(x_{i}\right)+x_{j, 2}\right)-\frac{E_{i} \dot{\eta}}{\eta}\right)\right)
\end{aligned}
$$

where $\xi_{i}=\frac{1}{2 \eta}\left(\frac{1}{\sigma+\delta_{\min }}-\frac{1}{\sigma-\delta_{\max }}\right)$.

\subsection{The Research of Radial Basis Function Neural}

Networks

In this paper, Radial Basis Function Neural Network$\mathrm{s}(\mathrm{RBFNNs})$ are introduced to approximate the unknown nonlinear functions $f_{i, l}\left(x_{i}\right)$ and $f_{i, n}\left(x_{i}\right)$ by

$f(x)=\theta^{* T} \varphi(x)+\tau(x)$, 
where $\varphi(x)=\left[\varphi_{1}(x), \varphi_{2}(x), \ldots, \varphi_{M}(x)\right]^{T}$ is the basis function vector. $\theta^{*}$ denotes the optimal weight vector. $M$ denotes the NN nodes' number, $M>1$. And approximate error $\tau(x)$ satisfies $|\tau(x)| \leqslant \varepsilon, \varepsilon>0$.

Further, the optimal weight vector $\theta^{*}$ and the Gaussian functions $\varphi_{i}(x)$ satisfy

$\theta^{*}=\arg \min _{\theta \in R^{M}}\left\{\sup _{x \in \Omega}\left|f(x)-\theta^{T} \varphi(x)\right|\right\}$,

$\varphi_{i}(x)=\exp \left(\frac{-\left(x-\mu_{i}\right)^{T}\left(x-\mu_{i}\right)}{\varpi_{i}^{2}}\right)$,

with $i=1,2, \ldots, M, \varpi_{i}$ and $\mu_{i}=\left[\mu_{i, 1}, \mu_{i, 2}, \ldots, \mu_{i, M}\right]^{T}$ are the width and the center of the Gaussian functions, respectively.

Lemma 5 [41]: Define $\varphi\left(\bar{x}_{q}\right)=\left[\varphi_{1}\left(\bar{x}_{q}\right), \ldots, \varphi_{M}\left(\bar{x}_{q}\right)\right]^{T}$, and $\bar{x}_{q}=\left[x_{1}, x_{2}, \ldots, x_{q}\right]^{T}$ is the basis function vector. Therefore, the following expression can be obtained, $\left\|\varphi\left(\bar{x}_{q}\right)\right\|^{2} \leqslant\left\|\varphi\left(\bar{x}_{p}\right)\right\|^{2}, p \leqslant q$.

\section{Event-triggered Prescribed Settling Time Consensus Control Design and Stability Analysis}

This paper's objective is to establish an event-triggered prescribed settling time consensus control method to guarantee that the synchronization error of MASs maintains the prescribed performance bounds, and the system can uniformly bounded convergent within the prescribed settling time.

3.1 Event-triggered Prescribed Settling Time Consensus Control Design

Firstly, the following transformation function is introduced to achieve the prescribed settling time tracking performance of MASs.

$\lambda_{i}(t)=\left\{\begin{array}{lr}\frac{T^{2} e^{\vartheta t}}{(1-\mu)(T-t)^{2}+\mu T^{2} e^{\vartheta t}}, 0 \leqslant t<T, \\ \frac{1}{\mu}, \quad t \geqslant T,\end{array}\right.$

where $\vartheta$ and $\mu$ are positive design constants, $0<\mu \ll 1$. $T$ denotes the practical prescribed settling time.

Remark 1: Note that $\lambda_{i}(t)$ is continuously differentiable, and $\dot{\lambda}_{i}(t)$ is bounded. From (14), it is obviously that $\lambda_{i}(t)$ is strictly monotonically increasing, $\lambda_{i}(0)=1, \lambda_{i}(t)=\frac{1}{\mu}$ for $t \geqslant T$.

Then, the following coordinate transformation will be performed.

$\left\{\begin{array}{l}z_{i, 1}=\lambda_{i} z_{i, 0}, i=1,2, \ldots, N, \\ z_{i, k}=x_{i, k}-\alpha_{i, k-1},\end{array}\right.$ where $k=2,3, \ldots, n$. And $\alpha$ is virtual control law.

Step 1: Construct the Lyapunov function $V_{i, 1}$ as

$V_{i, 1}=\frac{1}{2} z_{i, 1}^{2}+\frac{1}{2 \zeta_{i, 1}} \tilde{K}_{i, 1}^{T} \tilde{K}_{i, 1}$,

where $\zeta_{i, 1}>0$ is a designed parameter, $\tilde{K}_{i, 1}=K_{i, 1}$ $\hat{K}_{i, 1}$ denotes parameter estimation error.

Based on (10), (15) and (16), one has

$$
\begin{aligned}
\dot{V}_{i, 1}= & z_{i, 1} \dot{z}_{i, 1}-\frac{1}{\zeta_{i, 1}} \tilde{K}_{i, 1}^{T} \dot{\hat{K}}_{i, 1} \\
= & z_{i, 1}\left(\dot{\lambda}_{i} z_{i, 0}+\lambda_{i} \dot{z}_{i, 0}\right)-\frac{1}{\zeta_{i, 1}} \tilde{K}_{i, 1}^{T} \dot{\hat{K}}_{i, 1} \\
= & z_{i, 1}\left(\dot{\lambda}_{i} z_{i, 0}+\lambda_{i} \xi_{i}\left(\left(b_{i}+d_{i}\right)\left[f_{i, 1}\left(x_{i}\right)+x_{i, 2}\right]\right.\right. \\
& \left.\left.\quad-b_{i} \dot{y}_{d}-\sum_{j \in \mathcal{N}_{i}} a_{i j}\left(f_{j, 1}\left(x_{i}\right)+x_{j, 2}\right)-\frac{E_{i} \dot{\eta}}{\eta}\right)\right) \\
& \quad-\frac{1}{\zeta_{i, 1}} \tilde{K}_{i, 1}^{T} \dot{\hat{K}}_{i, 1} .
\end{aligned}
$$

According to the research of RBFNNs, define that

$$
\begin{aligned}
g_{i, 1}\left(x_{i}\right)= & \left(b_{i}+d_{i}\right) f_{i, 1}\left(x_{i}\right)-b_{i} \dot{y}_{d}-\sum_{j \in \mathcal{N}_{i}} a_{i j}\left(f_{j, 1}\left(x_{i}\right)+x_{j, 2}\right) \\
& \leqslant \theta_{i, 1}^{T} \varphi_{i, 1}+\varepsilon_{i, 1},
\end{aligned}
$$

Subsequently

$$
\begin{aligned}
\dot{V}_{i, 1} \leqslant & z_{i, 1}\left(\dot{\lambda}_{i} z_{i, 0}+\lambda_{i} \xi_{i}\left(\left(b_{i}+d_{i}\right) x_{i, 2}+\theta_{i, 1}^{T} \varphi_{i, 1}+\varepsilon_{i, 1}\right.\right. \\
& \left.\left.-\frac{E_{i} \dot{\eta}}{\eta}\right)\right)-\frac{1}{\zeta_{i, 1}} \tilde{K}_{i, 1}^{T} \dot{\hat{K}}_{i, 1} \\
\leqslant & z_{i, 1} \dot{\lambda}_{i} z_{i, 0}+z_{i, 1} \lambda_{i} \xi_{i}\left(b_{i}+d_{i}\right) z_{i, 2}+z_{i, 1} \lambda_{i} \xi_{i} \varepsilon_{i, 1} \\
& +z_{i, 1} \lambda_{i} \xi_{i} \theta_{i, 1}^{T} \varphi_{i, 1}+z_{i, 1} \lambda_{i} \xi_{i}\left(\left(b_{i}+d_{i}\right) \alpha_{1}-\frac{E_{i} \dot{\eta}}{\eta}\right) \\
& -\frac{1}{\zeta_{i, 1}} \tilde{K}_{i, 1}^{T} \dot{\hat{K}}_{i, 1} \\
\leqslant & z_{i, 1} \lambda_{i} \xi_{i}\left(\left(b_{i}+d_{i}\right) \alpha_{1}-\frac{E_{i} \dot{\eta}}{\eta}\right)-\frac{1}{\zeta_{i, 1}} \tilde{K}_{i, 1}^{T} \dot{\hat{K}}_{i, 1}+\Omega_{i, 1}
\end{aligned}
$$

where $\Omega_{i, 1}=z_{i, 1} \dot{\lambda}_{i} z_{i, 0}+z_{i, 1} \lambda_{i} \xi_{i}\left(b_{i}+d_{i}\right) z_{i, 2}+z_{i, 1} \lambda_{i} \xi_{i} \theta_{i, 1}^{T} \varphi_{i, 1}+$ $z_{i, 1} \lambda_{i} \xi_{i} \varepsilon_{i, 1}$.

By employing Lemma 2, we have

$$
\begin{aligned}
& z_{i, 1} \dot{\lambda}_{i} z_{i, 0} \leqslant \frac{z_{i, 1}^{2} z_{i, 0}^{2}}{2}+\frac{\dot{\lambda}_{i}^{2}}{2} \leqslant \frac{z_{i, 1}^{2} z_{i, 0}^{2}}{2}+\frac{\lambda_{i, \max }^{2}}{2} \\
& z_{i, 1} \lambda_{i} \xi_{i}\left(b_{i}+d_{i}\right) z_{i, 2} \leqslant \frac{z_{i, 1}^{2} \lambda_{i}^{2} \xi_{i}^{2}\left(b_{i}+d_{i}\right)^{2}}{2}+\frac{z_{i, 2}^{2}}{2},
\end{aligned}
$$


$z_{i, 1} \lambda_{i} \xi_{i} \theta_{i, 1}^{T} \varphi_{i, 1} \leqslant \frac{z_{i, 1}^{2} \lambda_{i}^{2} \xi_{i}^{2}\left\|\theta_{i, 1}\right\|^{2}\left\|\varphi_{i, 1}\right\|^{2}}{2 a_{i, 1}^{2}}+\frac{a_{i, 1}^{2}}{2}$,

$z_{i, 1} \lambda_{i} \xi_{i} \varepsilon_{i, 1} \leqslant \frac{z_{i, 1}^{2} \lambda_{i}^{2} \xi_{i}^{2}}{2}+\frac{\varepsilon_{i, 1}^{2}}{2}$,

where $a_{i, 1}$ is a designed parameter.

According to (20)-(23), one has

$$
\begin{aligned}
\Omega_{1} \leqslant & \frac{z_{i, 1}^{2} z_{i, 0}^{2}}{2}+\frac{z_{i, 1}^{2} \lambda_{i}^{2} \xi_{i}^{2}\left(b_{i}+d_{i}\right)^{2}}{2}+\frac{z_{i, 2}^{2}}{2}+\frac{z_{i, 1}^{2} \lambda_{i}^{2} \xi_{i}^{2}}{2} \\
& +\frac{z_{i, 1}^{2} \lambda_{i}^{2} \xi_{i}^{2}\left\|\theta_{i, 1}\right\|^{2}\left\|\varphi_{i, 1}\right\|^{2}}{2 a_{i, 1}^{2}}+\frac{\lambda_{i, \max }^{2}}{2}+\frac{a_{i, 1}^{2}}{2}+\frac{\varepsilon_{i, 1}^{2}}{2} \\
\leqslant & K_{i, 1} \varpi_{i, 1} z_{i, 1}^{2}+\frac{z_{i, 2}^{2}}{2}+\frac{\lambda_{i, \max }^{2}}{2}+\frac{a_{i, 1}^{2}}{2}+\frac{\varepsilon_{i, 1}^{2}}{2}
\end{aligned}
$$

where $K_{i, 1} \varpi_{i, 1} \geqslant \frac{z_{i, 0}^{2}}{2}+\lambda_{i}^{2} \xi_{i}^{2}\left(\frac{\left(b_{i}+d_{i}\right)^{2}}{2}+\frac{\left\|\theta_{i, 1}\right\|^{2}\left\|\varphi_{i, 1}\right\|^{2}}{2 a_{i, 1}^{2}}+\frac{1}{2}\right)$, $K_{i, 1}=\max \left\{\left(b_{i}+d_{i}\right)^{2},\left\|\theta_{i, 1}\right\|^{2}, 1\right\}$, and $\varpi_{i, 1}=\frac{z_{i, 0}^{2}}{2}+$ $\lambda_{i}^{2} \xi_{i}^{2}\left(1+\frac{\left\|\varphi_{i, 1}\right\|^{2}}{2 a_{i, 1}^{2}}\right)$.

Choose the virtual controller $\alpha_{i, 1}$ and adaptive law $\dot{\hat{K}}_{i, 1}$ as

$\alpha_{i, 1}=\frac{1}{b_{i}+d_{i}}\left(\frac{E_{i} \dot{\eta}}{\eta}-\frac{c_{i, 1} z_{i, 0}}{\xi_{i}}-\frac{\hat{K}_{i, 1} \varpi_{i, 1} z_{i, 0}}{\xi_{i}}\right)$,

$\dot{\hat{K}}_{i, 1}=\zeta_{i, 1} \varpi_{i, 1} z_{i, 1}^{2}-\chi_{i, 1} \hat{K}_{i, 1}$,

where $c_{i, 1}>0, \chi_{i, 1}>0$.

By substituting (24)-(26) into (19) yields

$$
\begin{aligned}
\dot{V}_{i, 1} \leqslant & z_{i, 1} \lambda_{i}\left(-c_{i, 1} z_{i, 0}-\hat{K}_{i, 1} \varpi_{i, 1} z_{i, 0}\right)-\frac{1}{\zeta_{i, 1}} \tilde{K}_{i, 1}^{T} \dot{\hat{K}}_{i, 1} \\
& +K_{i, 1} \varpi_{i, 1} z_{i, 1}^{2}+\frac{z_{i, 2}^{2}}{2}+\frac{\lambda_{i, \max }^{2}}{2}+\frac{a_{i, 1}^{2}}{2}+\frac{\varepsilon_{i, 1}^{2}}{2} \\
\leqslant & -c_{i, 1} z_{i, 1}^{2}-\hat{K}_{i, 1} \varpi_{i, 1} z_{i, 1}^{2}-\frac{1}{\zeta_{i, 1}} \tilde{K}_{i, 1}^{T} \dot{\hat{K}}_{i, 1} \\
& +K_{i, 1} \varpi_{i, 1} z_{i, 1}^{2}+\frac{z_{i, 2}^{2}}{2}+\Delta_{i, 1} \\
\leqslant & -c_{i, 1} z_{i, 1}^{2}+\frac{\chi_{i, 1} \tilde{K}_{i, 1}^{T} \hat{K}_{i, 1}}{\zeta_{i, 1}}+\frac{z_{i, 2}^{2}}{2}+\Delta_{i, 1}
\end{aligned}
$$

where $\Delta_{i, 1}=\frac{\lambda_{i, \max }^{2}}{2}+\frac{a_{i, 1}^{2}}{2}+\frac{\varepsilon_{i, 1}^{2}}{2}$.

Step 2: Construct the Lyapunov function $V_{i, 2}$ as

$$
V_{i, 2}=V_{i, 1}+\frac{1}{2} z_{i, 2}^{2}+\frac{1}{2 \zeta_{i, 2}} \tilde{K}_{i, 2}^{T} \tilde{K}_{i, 2}
$$

where $\zeta_{i, 2}>0$ is a designed parameter, $\tilde{K}_{i, 2}=K_{i, 2}-$ $\hat{K}_{i, 2}$ denotes parameter estimation error.
Combined (15) and (28) yields

$$
\begin{aligned}
\dot{V}_{i, 2}= & \dot{V}_{i, 1}+z_{i, 2} \dot{z}_{i, 2}-\frac{1}{\zeta_{i, 2}} \tilde{K}_{i, 2}^{T} \dot{\hat{K}}_{i, 2} \\
= & \dot{V}_{i, 1}+z_{i, 2}\left(z_{i, 3}+\alpha_{i, 2}+f_{i, 2}-\dot{\alpha}_{i, 1}\right) \\
& -\frac{1}{\zeta_{i, 2}} \tilde{K}_{i, 2}^{T} \dot{\hat{K}}_{i, 2} .
\end{aligned}
$$

According to the design of $\alpha_{i, 1}$, it can be obtained that

$$
\begin{aligned}
\dot{\alpha}_{i, 1}= & \frac{\partial \alpha_{i, 1}}{\partial x_{i, 1}} \dot{x}_{i, 1}+\frac{\partial \alpha_{i, 1}}{\partial x_{j, 1}} \dot{x}_{j, 1}+\frac{\partial \alpha_{i, 1}}{\partial y_{i, d}} \dot{y}_{i, d} \\
& +\frac{\partial \alpha_{i, 1}}{\partial \lambda_{i}} \dot{\lambda}_{i}+\frac{\partial \alpha_{i, 1}}{\partial \eta} \dot{\eta}+\frac{\partial \alpha_{i, 1}}{\partial \dot{\eta}} \ddot{\eta}+\frac{\partial \alpha_{i, 1}}{\partial \hat{K}_{i, 1}} \dot{\hat{K}}_{i, 1}
\end{aligned}
$$

From the research of RBFNNs, one has

$$
\begin{aligned}
g_{i, 2}\left(x_{i}\right)= & f_{i, 2}-\frac{\partial \alpha_{i, 1}}{\partial x_{i, 1}} \dot{x}_{i, 1}-\frac{\partial \alpha_{i, 1}}{\partial x_{j, 1}} \dot{x}_{j, 1}-\frac{\partial \alpha_{i, 1}}{\partial y_{i, d}} \dot{y}_{i, d} \\
& -\frac{\partial \alpha_{i, 1}}{\partial \lambda_{i}} \dot{\lambda}_{i}-\frac{\partial \alpha_{i, 1}}{\partial \eta} \dot{\eta}-\frac{\partial \alpha_{i, 1}}{\partial \dot{\eta}} \ddot{\eta} \\
\leqslant & \theta_{i, 2}^{T} \varphi_{i, 2}+\varepsilon_{i, 2}
\end{aligned}
$$

From (29), (30) and (31) yields

$$
\begin{aligned}
\dot{V}_{i, 2} \leqslant & -c_{i, 1} z_{i, 1}^{2}+\frac{\chi_{i, 1} \tilde{K}_{i, 1}^{T} \hat{K}_{i, 1}}{\zeta_{i, 1}}+\frac{z_{i, 2}^{2}}{2}+\Delta_{i, 1} \\
& +z_{i, 2}\left(z_{i, 3}+\alpha_{i, 2}+\theta_{i, 2}^{T} \varphi_{i, 2}+\varepsilon_{i, 2}-\frac{\partial \alpha_{i, 1}}{\partial \hat{K}_{i, 1}} \dot{\hat{K}}_{i, 1}\right) \\
& -\frac{1}{\zeta_{i, 2}} \tilde{K}_{i, 2}^{T} \dot{\hat{K}}_{i, 2} \\
\leqslant & -c_{i, 1} z_{i, 1}^{2}+\frac{\chi_{i, 1} \tilde{K}_{i, 1}^{T} \hat{K}_{i, 1}}{\zeta_{i, 1}}+\Delta_{i, 1}-\frac{1}{\zeta_{i, 2}} \tilde{K}_{i, 2}^{T} \dot{\hat{K}}_{i, 2} \\
& +z_{i, 2}\left(\frac{z_{i, 2}}{2}+\alpha_{i, 2}-\frac{\partial \alpha_{i, 1}}{\partial \hat{K}_{i, 1}} \dot{\hat{K}}_{i, 1}\right)+\Omega_{i, 2} .
\end{aligned}
$$

where $\Omega_{i, 2}=z_{i, 2} z_{i, 3}+z_{i, 2} \theta_{i, 2}^{T} \varphi_{i, 2}+z_{i, 2} \varepsilon_{i, 2}$.

Similarly, with the aid of Lemma $2, \Omega_{i, 2}$ can be transformed to

$$
\begin{aligned}
\Omega_{i, 2} & \leqslant \frac{z_{i, 2}^{2}}{2}+\frac{z_{i, 3}^{2}}{2}+\frac{z_{i, 2}^{2}\left\|\theta_{i, 2}\right\|^{2}\left\|\varphi_{i, 2}\right\|^{2}}{2 a_{i, 2}^{2}}+\frac{a_{i, 2}^{2}}{2}+\frac{z_{i, 2}^{2}}{2}+\frac{\varepsilon_{i, 2}^{2}}{2} \\
& \leqslant K_{i, 2} \varpi_{i, 2} z_{i, 2}^{2}+\frac{z_{i, 3}^{2}}{2}+\frac{a_{i, 2}^{2}}{2}+\frac{\varepsilon_{i, 2}^{2}}{2}
\end{aligned}
$$

where $\varpi_{i, 2}=1+\frac{\left\|\varphi_{i, 2}\right\|^{2}}{2 a_{i, 2}^{2}}, K_{i, 2}=\max \left\{1,\left\|\theta_{i, 2}\right\|^{2}\right\}$. 
Choose virtual controller $\alpha_{i, 2}$ and adaptive law $\dot{\hat{K}}_{i, 2}$ as

$$
\begin{aligned}
\alpha_{i, 2}= & -c_{i, 2} z_{i, 2}-\frac{1}{2} z_{i, 2}+\frac{\partial \alpha_{i, 1}}{\partial \hat{K}_{i, 1}} \dot{\hat{K}}_{i, 1} \\
& -\hat{K}_{i, 2} \varpi_{i, 2} z_{i, 2} \\
\dot{\hat{K}}_{i, 2}= & \zeta_{i, 2} \varpi_{i, 2} z_{i, 2}^{2}-\chi_{i, 2} \hat{K}_{i, 2},
\end{aligned}
$$

where $c_{i, 2}>0, \chi_{i, 2}>0$.

By substituting (33)-(35) into (32), we have

$$
\begin{aligned}
\dot{V}_{i, 2} \leqslant & -c_{i, 1} z_{i, 1}^{2}+\frac{\chi_{i, 1} \tilde{K}_{i, 1}^{T} \hat{K}_{i, 1}}{\zeta_{i, 1}}+K_{i, 2} \varpi_{i, 2} z_{i, 2}^{2} \\
& +\frac{z_{i, 3}^{2}}{2}+z_{i, 2}\left(\frac{z_{i, 2}}{2}+\alpha_{i, 2}-\frac{\partial \alpha_{i, 1}}{\partial \hat{K}_{i, 1}} \dot{K}_{i, 1}\right) \\
& +\frac{a_{i, 2}^{2}}{2}+\frac{\varepsilon_{i, 2}^{2}}{2}+\Delta_{i, 1}-\frac{1}{\zeta_{i, 2}} \tilde{K}_{i, 2}^{T} \dot{\hat{K}}_{i, 2} \\
\leqslant & -c_{i, 1} z_{i, 1}^{2}-c_{i, 2} z_{i, 2}^{2}+\frac{z_{i, 3}^{2}}{2}+\frac{\chi_{i, 1} \tilde{K}_{i, 1}^{T} \hat{K}_{i, 1}}{\zeta_{i, 1}} \\
& -\hat{K}_{i, 2} \varpi_{2} z_{i, 2}^{2}+K_{i, 2} \varpi_{i, 2} z_{i, 2}^{2}+\Delta_{i, 2} \\
& -\frac{1}{\zeta_{i, 2}} \tilde{K}_{i, 2}^{T}\left(\zeta_{i, 2} \varpi_{2} z_{i, 2}^{2}-\xi_{i, 2} \hat{K}_{i, 2}\right) \\
\leqslant & -c_{i, 1} z_{i, 1}^{2}-c_{i, 2} z_{i, 2}^{2}+\frac{z_{i, 3}^{2}}{2}+\frac{\chi_{i, 1} \tilde{K}_{i, 1}^{T} \hat{K}_{i, 1}}{\zeta_{i, 1}} \\
& +\frac{\chi_{i, 2} \tilde{K}_{i, 2}^{T} \hat{K}_{i, 2}}{\zeta_{i, 2}}+\Delta_{i, 2},
\end{aligned}
$$

where $\Delta_{i, 2}=\Delta_{i, 1}+\frac{a_{i, 2}^{2}}{2}+\frac{\varepsilon_{i, 2}^{2}}{2}$.

Step $k(k=3,4, \ldots, n-1)$ : Construct the Lyapunov function $V_{i, k}$ as

$$
V_{i, k}=V_{i, k}+\frac{1}{2} z_{i, k}^{2}+\frac{1}{2 \zeta_{i, k}} \tilde{K}_{i, k}^{T} \tilde{K}_{i, k}
$$

where $\zeta_{i, k}>0, \tilde{K}_{i, k}=K_{i, k}-\hat{K}_{i, k}$ denotes parameter error.

From (15) and (37), we have

$$
\begin{aligned}
\dot{V}_{i, k}= & \dot{V}_{i, k-1}+z_{i, k}\left(z_{i, k+1}+\alpha_{i, k}+f_{i, k}-\dot{\alpha}_{i, k-1}\right) \\
& -\frac{1}{\zeta_{i, k}} \tilde{K}_{i, k}^{T} \dot{\hat{K}}_{i, k}
\end{aligned}
$$

Similar to (30), it follows

$$
\begin{aligned}
\dot{\alpha}_{i, k-1}= & \sum_{l=1}^{k-1} \frac{\partial \alpha_{i, k-1}}{\partial x_{i, l}} \dot{x}_{i, l}+\sum_{l=1}^{k-1} \frac{\partial \alpha_{i, k-1}}{\partial x_{j, l}} \dot{x}_{j, l} \\
& +\frac{\partial \alpha_{i, k-1}}{\partial y_{i, d}} \dot{y}_{i, d}+\sum_{l=1}^{k-1} \frac{\partial \alpha_{i, k-1}}{\partial \hat{K}_{i, l}} \dot{\hat{K}}_{i, l}
\end{aligned}
$$

From the research of RBFNNs, one has

$$
\begin{aligned}
g_{i, k}\left(x_{i}\right)= & f_{i, k}-\sum_{l=1}^{k-1} \frac{\partial \alpha_{i, k-1}}{\partial x_{i, l}} \dot{x}_{i, l}-\frac{\partial \alpha_{i, k-1}}{\partial y_{i, d}} \dot{y}_{i, d} \\
& -\sum_{l=1}^{k-1} \frac{\partial \alpha_{i, k-1}}{\partial x_{j, l}} \dot{x}_{j, l} \\
\leqslant & \theta_{i, k}^{T} \varphi_{i, k}+\varepsilon_{i, k} .
\end{aligned}
$$

With the aid of (39) and (40), (38) can be transformed to

$$
\begin{aligned}
\dot{V}_{i, k} \leqslant & -\sum_{l=1}^{k-1} c_{i, k-1} z_{i, k-1}^{2}+\sum_{l=1}^{k-1} \frac{\chi_{i, l} \tilde{K}_{i, l}^{T} \hat{K}_{i, l}}{\zeta_{i, l}}+\frac{z_{i, k}^{2}}{2} \\
& +\Delta_{i, k-1}-\frac{1}{\zeta_{i, k}} \tilde{K}_{i, k}^{T} \dot{\hat{K}}_{i, k}+z_{i, k}\left(z_{i, k+1}+\alpha_{i, k}\right. \\
& \left.+\theta_{i, k}^{T} \phi_{i, k}+\varepsilon_{i, k}-\sum_{l=1}^{k-1} \frac{\partial \alpha_{i, k-1}}{\partial \hat{\hat{K}}_{i, l}}\right) \\
\leqslant & -\sum_{l=1}^{k-1} c_{i, k-1} z_{i, k-1}^{2}+\sum_{l=1}^{k-1} \frac{\chi_{i, l} \tilde{K}_{i, l}^{T} \hat{K}_{i, l}}{\zeta_{i, l}}+\Delta_{i, k-1} \\
& -\frac{1}{\zeta_{i, k}} \tilde{K}_{i, k}^{T} \dot{\hat{K}}_{i, k}+z_{i, k}\left(\frac{z_{i, k}}{2}+\alpha_{i, k}\right. \\
& \left.-\sum_{l=1}^{k-1} \frac{\partial \alpha_{i, k-1}}{\partial \hat{K}_{i, l}} \dot{\hat{K}}_{i, l}\right)+\Omega_{i, k},
\end{aligned}
$$

where $\Omega_{i, k}=z_{i, k} z_{i, k+1}+\theta_{i, k}^{T} \varphi_{i, k} z_{i, k}+\varepsilon_{i, k} z_{i, k}$.

According to Lemma $2, \Omega_{i, k}$ can be rewritten to

$$
\begin{aligned}
\Omega_{i, k} \leq & \frac{z_{i, k}^{2}}{2}+\frac{z_{i, k+1}^{2}}{2}+\frac{z_{i, k}^{2}\left\|\theta_{i, k}\right\|^{2}\left\|\varphi_{i, k}\right\|^{2}}{2 a_{i, k}^{2}} \\
& +\frac{a_{i, k}^{2}}{2}+\frac{z_{i, k}^{2}}{2}+\frac{\varepsilon_{i, k}^{2}}{2} \\
\leq & \varpi_{i, k} K_{i, k} z_{i, k}^{2}+\frac{z_{i, k+1}^{2}}{2}+\frac{a_{i, k}^{2}}{2}+\frac{\varepsilon_{i, k}^{2}}{2},
\end{aligned}
$$

where $\varpi_{i, k}=1+\frac{\left\|\theta_{i, k}\right\|^{2}}{2 a_{i, k}^{2}}, K_{i, k}=\max \left\{1,\left\|\varphi_{i, k}\right\|^{2}\right\}$.

Choose the virtual controller $\alpha_{i, k}$ and adaptive law $\dot{\hat{K}}_{i, k}$ as

$$
\begin{aligned}
& \alpha_{i, k}=-c_{i, k} z_{i, k}-\frac{1}{2} z_{i, k}+\sum_{l=1}^{k-1} \frac{\partial \alpha_{i, k-1}}{\partial \hat{K}_{i, l}} \dot{\hat{K}}_{i, l} \\
&-\hat{K}_{i, k} \varpi_{i, k} z_{i, k}, \\
& \dot{\hat{K}}_{i, k}= \zeta_{i, k} \varpi_{i, k} z_{i, k}^{2}-\chi_{i, k} \hat{K}_{i, k}, \\
& \text { where } c_{i, k}>0, \chi_{i, k}>0 .
\end{aligned}
$$


By substituting (42)-(44) into (41) yields

$$
\begin{aligned}
\dot{V}_{i, k} \leqslant & -\sum_{l=1}^{k-1} c_{k-1} z_{i, k-1}^{2}+\sum_{l=1}^{k-1} \frac{\chi_{i, l} \tilde{K}_{i, l}^{T} \hat{K}_{i, l}}{\zeta_{i, l}}+\Delta_{i, k-1} \\
& +z_{i, k}\left(\frac{z_{i, k}}{2}+\alpha_{i, k}-\sum_{l=1}^{k-1} \frac{\partial \alpha_{i, k-1}}{\partial \hat{K}_{i, l}}\right)+\frac{z_{i, k+1}^{2}}{2} \\
& +z_{i, k}^{2} \varpi_{i, k} K_{i, k}+\frac{a_{i, k}^{2}}{2}+\frac{\varepsilon_{i, k}^{2}}{2}-\frac{1}{\zeta_{i, k}} \tilde{K}_{i, k}^{T} \dot{\hat{K}}_{i, k} \\
\leqslant & -\sum_{l=1}^{k} c_{i, l} z_{i, l}^{2}+\frac{z_{i, k+1}^{2}}{2}+\sum_{l=1}^{k-1} \frac{\chi_{i, l} \tilde{K}_{i, l}^{T} \hat{K}_{i, l}}{\zeta_{i, l}}+\Delta_{i, k} \\
& -\hat{K}_{i, k} \varpi_{k} z_{i, k}^{2}+z_{i, k}^{2} \varpi_{i, k} K_{i, k}-\frac{1}{\zeta_{i, k}} \tilde{K}_{i, k}^{T} \dot{\hat{K}}_{i, k} \\
\leqslant & -\sum_{l=1}^{k} c_{i, l} z_{i, l}^{2}+\frac{z_{i, k+1}^{2}}{2}+\sum_{l=1}^{k} \frac{\chi_{i, l} \tilde{K}_{i, l}^{T} \hat{K}_{i, l}}{\zeta_{i, l}}+\Delta_{i, k}
\end{aligned}
$$

where $\Delta_{i, k}=\Delta_{i, k-1}+\frac{a_{i, k}^{2}}{2}+\frac{\varepsilon_{i, k}^{2}}{2}$.

Step $\boldsymbol{n}$ : The event-triggered control mechanism and adaptive law $\dot{\hat{K}}_{i, n}$ are given.

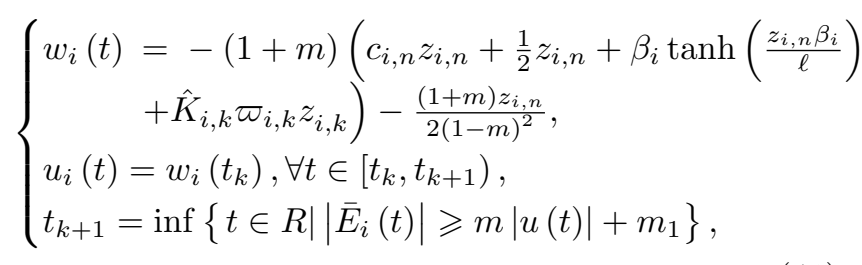

$\dot{\hat{K}}_{i, n}=\zeta_{i, n} \varpi_{n} z_{i, n}^{2}-\chi_{i, n} \hat{K}_{i, n}$

where measurement error is $\bar{E}_{i}(t)=w_{i}(t)-u_{i}(t)$, $0<m<1$, $\ell$ and $m_{1}$ are positive designed parameters, $\hat{K}_{i, n}(0) \geqslant 0$. Moreover, $\beta_{i}=\sum_{l=1}^{n-1} \frac{\partial \alpha_{i, n-1}}{\partial \hat{K}_{i, l}} \dot{\hat{K}}_{i, l}$.

Remark 2: Since the threshold value of the eventtriggered mechanism used in this paper changes with the change of the control signal, the term $m_{1}$ is mainly used to control the trigger interval and avoid Zenobehavior. When $m_{1}$ increases, the trigger interval becomes longer and the required communication resources decrease. However, due to the slow update of the system control signal, the control performance may decrease. When M decreases, the trigger interval becomes shorter and the required communication resources increase. However, because the system control signal is updated faster, the control performance will be better. Therefore, selecting moderate event trigger parameters $m_{1}$ plays an important role in balancing system control performance and communication resources.
Construct the Lyapunov function $V_{i, n}$ as

$V_{i, n}=V_{i, n}+\frac{1}{2} z_{i, n}^{2}+\frac{1}{2 \zeta_{i, n}} \tilde{K}_{i, n}^{T} \tilde{K}_{i, n}$,

where $\zeta_{i, n}>0, \tilde{K}_{i, n}=K_{i, n}-\hat{K}_{i, n}$ is parameter estimation error.

From (15) and (48), we have

$$
\begin{aligned}
\dot{V}_{i, n} & =\dot{V}_{i, n-1}+z_{i, n} \dot{z}_{i, n}-\frac{1}{\zeta_{i, n}} \tilde{K}_{i, n}^{T} \dot{\hat{K}}_{i, n} \\
& =\dot{V}_{i, n-1}+z_{i, n}\left(u_{i}+f_{i, n}-\dot{\alpha}_{i, n-1}\right)-\frac{1}{\zeta_{i, n}} \tilde{K}_{i, n}^{T} \dot{\hat{K}}_{i, n}
\end{aligned}
$$

Similar to (39), (50) can be obtained.

$$
\begin{aligned}
\dot{\alpha}_{i, n-1}= & \sum_{l=1}^{n-1} \frac{\partial \alpha_{i, n-1}}{\partial x_{i, l}} \dot{x}_{i, l}+\sum_{l=1}^{n-1} \frac{\partial \alpha_{i, n-1}}{\partial x_{j, l}} \dot{x}_{j, l} \\
& +\frac{\partial \alpha_{i, n-1}}{\partial y_{i, d}} \dot{y}_{i, d}+\sum_{l=1}^{n-1} \frac{\partial \alpha_{i, n-1}}{\partial \hat{K}_{i, l}} \dot{\hat{K}}_{i, l}
\end{aligned}
$$

From the definition of the event-triggered control mechanism mentioned earlier, one has

$w_{i}(t)=\left(1+\kappa_{1}(t) m\right) u(t)+\kappa_{2}(t) m_{1}$,

where $\left|\kappa_{1}(t)\right| \leqslant 1$ and $\left|\kappa_{2}(t)\right| \leqslant 1$ are time-varying parameters.

Subsequently

$$
\begin{aligned}
\dot{V}_{i, n}= & \dot{V}_{i, n-1}+z_{i, n}\left(u_{i}+f_{i, n}-\sum_{l=1}^{n-1} \frac{\partial \alpha_{i, n-1}}{\partial x_{i, l}} \dot{x}_{i, l}\right. \\
& \left.-\sum_{l=1}^{n-1} \frac{\partial \alpha_{i, n-1}}{\partial x_{j, l}} \dot{x}_{j, l}-\frac{\partial \alpha_{i, n-1}}{\partial y_{i, d}} \dot{y}_{i, d}+\beta_{i}\right) \\
& -\frac{1}{\zeta_{i, n}} \tilde{K}_{i, n}^{T} \dot{\hat{K}}_{i, n} \\
= & -\sum_{l=1}^{n-1} c_{i, l} z_{i, l}^{2}+\frac{z_{i, n}^{2}}{2}+\sum_{l=1}^{n-1} \frac{\chi_{i, l} \tilde{K}_{i, l}^{T} \hat{K}_{i, l}}{\zeta_{i, l}} \\
& +\Delta_{i, n-1}+z_{i, n} \beta_{i}+\frac{z_{i, n} w_{i}(t)}{1+\kappa_{1}(t) m} \\
& -\frac{z_{i, n} \kappa_{2}(t) m_{1}}{1+\kappa_{1}(t) m}+\Omega_{i, n}-\frac{1}{\zeta_{i, n}} \tilde{K}_{i, n}^{T} \dot{\hat{K}}_{i, n}
\end{aligned}
$$

where $\Omega_{i, n}=z_{i, n} \theta_{i, n}^{T} \varphi_{i, n}+z_{i, n} \varepsilon_{i, n}$.

From Lemma 2, $\Omega_{i, n}$ can be transformed to

$$
\begin{aligned}
\Omega_{i, n} & \leqslant \frac{z_{i, n}^{2}\left\|\theta_{i, n}\right\|^{2}\left\|\phi_{i, n}\right\|^{2}}{2 a_{i, n}^{2}}+\frac{z_{i, n}^{2}}{2}+\frac{a_{i, n}^{2}}{2}+\frac{\varepsilon_{i, n}^{2}}{2} \\
& \leqslant z_{i, n}^{2}\left(\frac{1}{2}+\frac{\left\|\theta_{i, n}\right\|^{2}\left\|\varphi_{i, n}\right\|^{2}}{2 a_{i, n}^{2}}\right)+\frac{a_{i, n}^{2}}{2}+\frac{\varepsilon_{i, n}^{2}}{2} \\
& \leqslant z_{i, n}^{2} \varpi_{i, n} K_{i, n}+\frac{a_{i, n}^{2}}{2}+\frac{\varepsilon_{i, n}^{2}}{2}
\end{aligned}
$$


where $\varpi_{i, n}=1+\frac{\left\|\varphi_{i, n}\right\|^{2}}{2 a_{i, n}^{2}}, K_{i, n}=\max \left\{\frac{1}{2},\left\|\theta_{i, n}\right\|^{2}\right\}$.

From the research of RBFNNs yields

$$
\begin{aligned}
g_{i, \mathrm{n}}\left(x_{i}\right)= & f_{i, n}-\sum_{l=1}^{n-1} \frac{\partial \alpha_{i, n-1}}{\partial x_{i, l}} \dot{x}_{i, l}-\sum_{l=1}^{n-1} \frac{\partial \alpha_{i, n-1}}{\partial x_{j, l}} \dot{x}_{j, l} \\
& -\frac{\partial \alpha_{i, n-1}}{\partial \lambda_{i}} \dot{\lambda}_{i}-\frac{\partial \alpha_{i, n-1}}{\partial y_{i, d}} \dot{y}_{i, d} \\
& \leqslant \theta_{i, n}^{T} \varphi_{i, n}+\varepsilon_{i, n} .
\end{aligned}
$$

Combined with (53), (54), (52) can be rewritten as

$$
\begin{aligned}
\dot{V}_{i, n} \leqslant & -\sum_{l=1}^{n-1} c_{l} z_{i, l}^{2}+\frac{z_{i, n}^{2}}{2}+\sum_{l=1}^{n-1} \frac{\chi_{i, l} \tilde{K}_{i, l}^{T} \hat{K}_{i, l}}{\zeta_{i, l}}+\Delta_{i, n-1} \\
& +\frac{z_{i, n} w_{i}(t)}{1+\kappa_{1}(t) m}-\frac{z_{i, n} \kappa_{2}(t) m_{1}}{1+\kappa_{1}(t) m}+z_{i, n}^{2} \varpi_{i, n} K_{i, n} \\
& +z_{i, n} \beta_{i}+\frac{a_{i, n}^{2}}{2}+\frac{\varepsilon_{i, n}^{2}}{2}-\frac{1}{\zeta_{i, n}} \tilde{K}_{i, n}^{T} \dot{\hat{K}}_{i, n} \\
\leqslant & -\sum_{l=1}^{n-1} c_{i, l} z_{i, l}^{2}+\frac{z_{i, n}^{2}}{2}+\sum_{l=1}^{n-1} \frac{\chi_{i, l} \tilde{K}_{i, l}^{T} \hat{K}_{i, l}}{\zeta_{i, l}}+\Delta_{i, n} \\
& +\frac{z_{i, n} w_{i}(t)}{1+\kappa_{1}(t) m}-\frac{z_{i, n} \kappa_{2}(t) m_{1}}{1+\kappa_{1}(t) m}+z_{i, n} \beta_{i} \\
& +z_{i, n}^{2} \varpi_{i, n} K_{i, n}-\frac{1}{\zeta_{i, n}} \tilde{K}_{i, n}^{T} \dot{\hat{K}}_{i, n},
\end{aligned}
$$

where $\Delta_{i, n}=\Delta_{i, n-1}+\frac{a_{i, n}^{2}}{2}+\frac{\varepsilon_{i, n}^{2}}{2}$.

According to the definition of (46) and (47) yields $z_{i, n} w_{i}(t) \leqslant 0$. Hence,

$$
\begin{aligned}
& \frac{z_{i, n} w_{i}(t)}{1+\kappa_{1}(t) m} \\
\leqslant & \frac{z_{i, n} w_{i}(t)}{1+m} \\
\leqslant & -z_{i, n}\left(c_{i, n} z_{i, n}+\frac{1}{2} z_{i, n}+\hat{K}_{i, n} \varpi_{n} z_{i, n}\right. \\
& \left.+\beta_{i} \tanh \left(\frac{z_{i, n} \beta_{i}}{\ell}\right)\right)-\frac{z_{i, n}^{2}}{2(1-m)^{2}} . \\
& -\frac{z_{i, n} \kappa_{2}(t) m_{1}}{1+\kappa_{1}(t) m} \leqslant \frac{\left|z_{i, n}\right| m_{1}}{1-m} \leqslant \frac{z_{i, n}^{2}}{2(1-m)^{2}}+\frac{m_{1}^{2}(t)}{2} .
\end{aligned}
$$

Remark 3: Note that $\bar{E}_{i}(t)$ depends on system input $w_{i}(t)$ and $u_{i}(t)$. Before establishing the stability of the system, $\bar{E}_{i}(t)$ cannot be assumed to be bounded, which brings difficulties to the design of the controller. To handle this issue, as long as the controller satisfies this property $z_{i, n} w_{i}(t) \leqslant 0$. Since $\forall \beta \in R, \tau>0$, then $-\beta \tanh \left(\frac{\beta}{\tau}\right) \leqslant 0$. Since $w_{i}(t), \hat{K}_{i, k}$ and $\varpi_{i, k}$, it can be obtained that $z_{i, n} w_{i}(t) \leqslant 0$. Thus, (56) holds.

By employing Lemma 2, one has

$$
z_{i, n} \beta_{i} \leqslant\left|z_{i, n} \beta_{i}\right| \leqslant z_{i, n} \beta_{i} \tanh \left(\frac{z_{i, n} \beta_{i}}{\ell}\right)+0.2785 \ell .
$$

Then, $\dot{V}_{i, n}$ can be further transformed to

$$
\begin{aligned}
\dot{V}_{i, n} \leqslant & -\sum_{l=1}^{n-1} c_{i, l} z_{i, l}^{2}+\frac{z_{i, n}^{2}}{2}+\sum_{l=1}^{n-1} \frac{\chi_{i, l} \tilde{K}_{i, l}^{T} \hat{K}_{i, l}}{\zeta_{i, l}}+\frac{m_{1}^{2}(t)}{2} \\
& -\frac{z_{i, n}^{2}}{2(1-m)^{2}}+\frac{z_{i, n}^{2}}{2(1-m)^{2}}-z_{i, n}\left(c_{i, n} z_{i, n}\right. \\
& \left.\quad+\frac{1}{2} z_{i, n}+\beta_{i} \tanh \left(\frac{z_{i, n} \beta_{i}}{\ell}\right)+\hat{K}_{i, n} \varpi_{i, n} z_{i, n}\right) \\
& +z_{i, n}^{2} \varpi_{i, n} K_{i, n}+z_{i, n} \beta_{i} \tanh \left(\frac{z_{i, n} \beta_{i}}{\ell}\right) \\
& +0.2785 \ell-\frac{1}{\zeta_{i, n}} \tilde{K}_{i, n}^{T} \dot{\hat{K}}_{i, n}+\Delta_{i, n} \\
\leqslant & -\sum_{l=1}^{n} c_{i, l} z_{i, l}^{2}+\sum_{l=1}^{n} \frac{\chi_{i, l} \tilde{K}_{i, l}^{T} \hat{K}_{i, l}}{\zeta_{i, l}}+\frac{m_{1}^{2}(t)}{2} \\
& +0.2785 \ell+\Delta_{i, n} \\
\leqslant & -\sum_{l=1}^{n} c_{i, l} z_{i, l}^{2}-\sum_{l=1}^{n} \frac{\chi_{i, n} \tilde{K}_{i, l}^{T} \tilde{K}_{i, l}}{2 \zeta_{i, l}}+\Delta_{i} \\
\leqslant & -\gamma V_{i, n}+\Delta_{i},
\end{aligned}
$$

where $\gamma=\min \left\{2 c_{i, 1}, 2 c_{i, 2}, \ldots, 2 c_{i, n}, 2 \chi_{i, 1}, 2 \chi_{i, 2}, \ldots, 2 \chi_{i, n}\right\}>$ $0, \Delta_{i, n}=\Delta_{i, n-1}+\frac{a_{i, n}^{2}}{2}+\frac{\varepsilon_{i, n}^{2}}{2}, \Delta_{i}=\sum_{l=1}^{n} \frac{\chi_{i, l} K_{i, l}^{2}}{2 \zeta_{i, l}}+\frac{m_{1}^{2}(t)}{2}+$ $0.2785 \ell+\Delta_{i, n}$.

\subsection{Stability analysis}

Theorem 1: The multiagent systems (1), the virtual controllers (25), (34), (43), the parameter update law (26), (35), (44), (47) and the event-triggered prescribed settling time consensus controller (46) are considered. The following result can be guaranteed.

1) All the signals of multiagent systems are bounded.

2) There is a time $t^{*}>0$ so that the inter-execution intervals $\left\{t_{k+1}-t_{k}\right\}$ are lower bounded by $t^{*}, \forall k \in$ $z^{+}$. Thus, Zeno-behavior can be avoided.

Proof: Choose the Lyapunov function $V$ as

$V=\sum_{i=1}^{N} V_{i, n}$ 
With the help of the design and derivation in the previous section, it has

$\dot{V} \leqslant \sum_{i=1}^{N}\left(-\gamma V_{i, n}+\Delta_{i}\right)$.

Thus

$\dot{V} \leqslant-\gamma V+\Delta$

where $\Delta=\sum_{i=1}^{N} \Delta_{i}$.

Combined with the analysis of [30], it can further obtain that

$\frac{1}{2} z_{i, 1}^{2} \leqslant V(t) \leqslant e^{-\gamma t}\left(V(0)-\frac{\Delta}{\gamma}\right)+\frac{\Delta}{\gamma}$,

When $V(0)>\frac{\Delta}{\gamma}, e^{-\gamma t}\left(V(0)-\frac{\Delta}{\gamma}\right)+\frac{\Delta}{\gamma} \leqslant V(0)$ holds. When $V(0) \leqslant \frac{\Delta}{\gamma}, e^{-\gamma t}\left(V(0)-\frac{\Delta}{\gamma}\right)+\frac{\Delta}{\gamma} \leqslant \frac{\Delta}{\gamma}$ holds. Define $\Theta=\max \left\{V(0), \frac{\Delta}{\gamma}\right\}, e^{-\gamma t}\left(V(0)-\frac{\Delta}{\gamma}\right)+$ $\frac{\Delta}{\gamma} \leqslant \Theta$ is always established, that is, $\left|z_{i, 1}\right| \leqslant \sqrt{2 \Theta}$.

Therefore, Choosing appropriate design parameters can guarantee MASs uniformly bounded convergent. Therefore, $z_{i, k}, \tilde{K}_{i, k}$ are bounded, $i=1,2, \ldots, N, k=$ $1,2, \ldots, n$. Since $\lambda_{i}, \eta(t)$, and $\rho\left(z_{i, 0}\right)$ are bounded function, $z_{i, 1}=\lambda_{i} z_{i, 0}, \tilde{K}_{i, k}=K_{i, k}-\hat{K}_{i, k}$. It implies that synchronization error $z_{i, 0}$, system states $x_{1,1}, x_{2,1}, \ldots, x_{N, 1}$ and $\hat{K}_{i, k}$ are also bounded. Then, the virtual control law $\alpha_{i, 1}$ contains the bounded signals $x_{i, 1}, x_{j, 1}$ and $\hat{K}_{i, k}$, it is obviously that $\alpha_{i, 1}$ is bounded too. By analogy, all signals of the MASs are bounded. Besides, applying to Lemma 3 and Lemma 4, it can draw the conclusion that the tracking error satisfies $\lim _{t \rightarrow \infty}\left\|y-Y_{d}\right\| \leqslant \frac{\sqrt{2 N \Theta}}{\vartheta}$.

According to the event-triggered strategy $(46), \bar{E}_{i}(t)=$ $w_{i}(t)-u_{i}(t)$, there will be a $t^{*}$ satisfies $\left\{t_{k+1}-t_{k}\right\} \geqslant$ $t^{*}, \forall \in z^{+}$.

Then

$\frac{d}{d t}\left|\bar{E}_{i}\right|=\frac{d}{d t}\left(\bar{E}_{i} \times \bar{E}_{i}\right)^{\frac{1}{2}}=\operatorname{sign}\left(\bar{e}_{i}\right) \dot{\bar{E}}_{i} \leqslant\left|w_{i}(t)\right|$.

Note that $f_{i, k}(\cdot)$ is nonlinear smooth function, it can be obtained that $w_{i}(t)$ is a nonlinear smooth function too. Since $w_{i}(t)$ is a function, which contains the bounded signals $z_{i, k}, \hat{K}_{i, k}$, so the time derivative of $w_{i}(t)$ will satisfy $\left|\dot{w}_{i}\right| \leqslant \varpi, \varpi>0$. Then, there will be $t^{*} \geqslant \frac{\max \left\{m, m_{1}\right\}}{\omega}$, where $t^{*}$ is the lower bound of time interval. Obviously, the considered system can effectively avoid Zeno-behavior.

According to (15), it can be obtained that $\left|z_{i, 0}\right|=$ $\lambda_{i}^{-1}\left|z_{i, 1}\right| \leqslant \lambda_{i}^{-1} \sqrt{2 \Theta}$. Combined with the definition in (14), it can easy arrive that when $0 \leqslant t<T,\left|z_{i, 0}\right| \leqslant$
$(1-\mu)\left(\frac{T-t}{T}\right)^{2} e^{-\vartheta t} \sqrt{2 \Theta}+\mu \sqrt{2 \Theta}$, when $t \geqslant T,\left|z_{i, 0}\right| \leqslant$ $\mu \sqrt{2 \Theta}$. It implies that the synchronization error of MASs converges to the compact set $\Omega_{i}=\left\{z_{i, 0}|| z_{i, 0} \mid \leqslant \mu \sqrt{2 \Theta}\right\}$ within the prescribed settling time $\mathrm{T}$. And its decay rate in not less that $\left(\frac{T-t}{T}\right)^{2} e^{-\vartheta t}$.

Remark 4: From the analysis of the prescribed settling time $T$, we know that the design of $T, \vartheta, \mu$ can influence the tracking performance of MASs. By adjusting the above parameters, better tracking performance can be obtained. But it will also have a certain impact on the transient performance of MASs at the same time. How to balance their relationship is a problem worthy of our attention.

\section{Illustrative Results}

Numerical example and practical example are carried out to verify the validity of the proposed event-triggered prescribed settling time consensus control method.

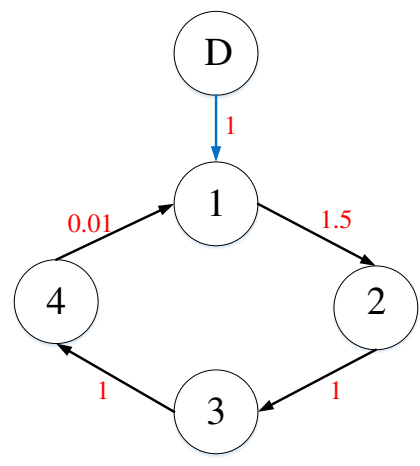

Fig. 1: Topology of communication graph.

\subsection{Numerical Example}

Consider the MASs shown in Fig 1. The model of the $i t h$ follower is presented.

$\left\{\begin{array}{l}\dot{x}_{i, 1}=x_{i, 2}+f_{i, 1}\left(x_{i, 1}\right), \\ \dot{x}_{i, 2}=u_{i}+f_{i, 2}\left(x_{i, 2}\right), \\ y_{i}=x_{i, 1}\end{array}\right.$

where $i=1,2,3,4, f_{i, 1}\left(x_{i, 1}\right)=0.2 \sin \left(x_{i, 1}\right), f_{i, 2}\left(x_{i, 2}\right)=$ $0.2 \sin \left(x_{i, 1}^{2} x_{i, 2}\right)$. The reference signal is $y_{d}=\sin t$. The event-triggered prescribed settling time consensus control proposed in this study will be applied to the above system. The system design parameters and initializations are presented in Table 1. 


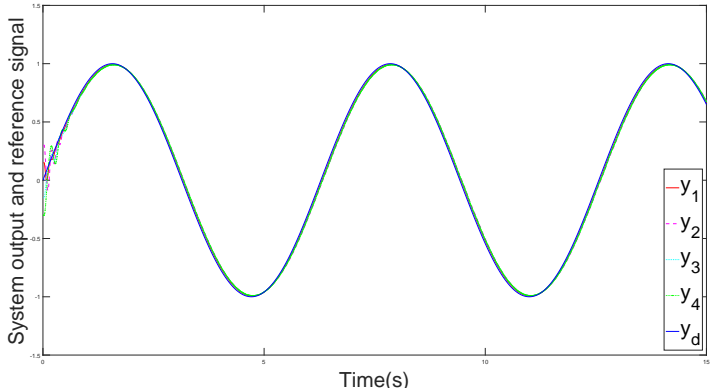

(a)

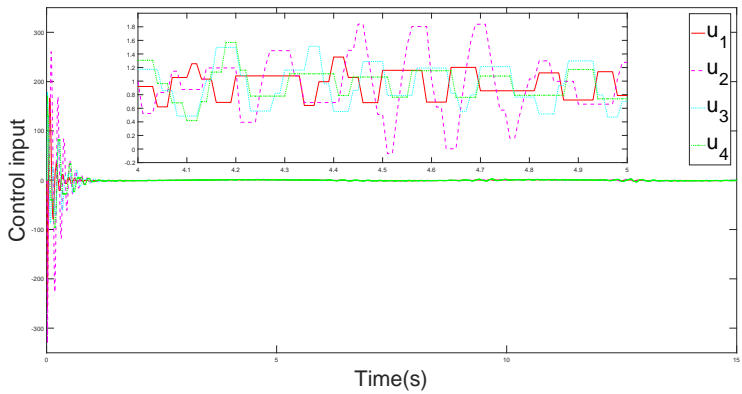

(c)

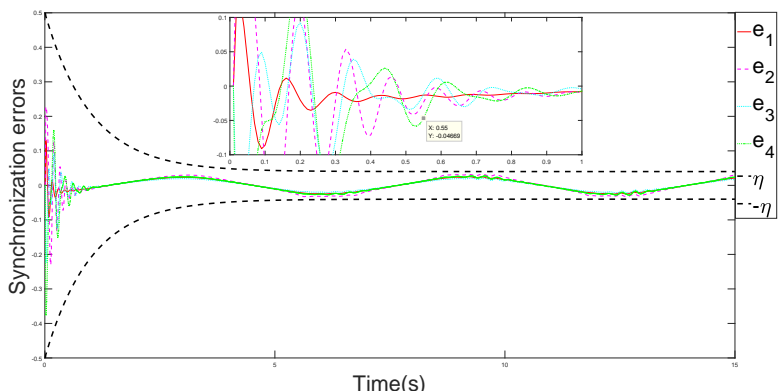

(b)
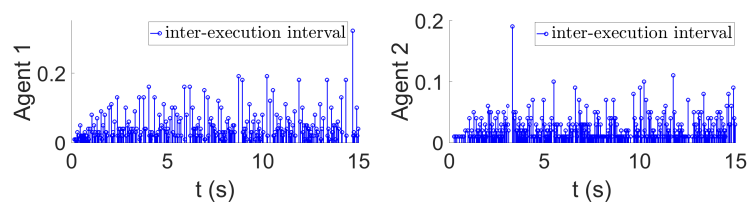

$\mathrm{t}(\mathrm{s})$

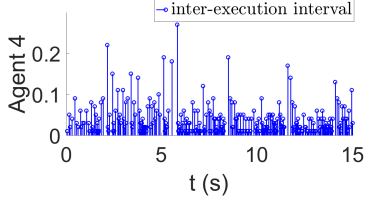

(d)

Fig. 2: Numerical example: (a) $y$ and $y_{d}$; (b) Synchronization error signal; (c) System event-triggered inputs; (d) Inter-execution interval

Table 1: The parameter of the proposed method

\begin{tabular}{|llllll|}
\hline$c_{1}=[48,60,55,45]^{T}$ & $c_{2}=[35,35,15,15]^{T}$ & $a_{1}=5$ & $a_{2}=5$ & $\varsigma_{1}=1$ & $\varsigma_{2}=2$ \\
$\chi_{i, 1}=10$ & $\chi_{i, 2}=10$ & $m=0.2$ & $\ell=0.9$ & $n=0.18$ & $m_{1}=0.1$ \\
$T=0.8$ & $\vartheta=0.5$ & $\mu=0.4$ & $\lambda(1)=0$ & $\delta_{\max }=1$ & $\delta_{\min }=0.999$ \\
$\eta_{0}=0.5$ & $\gamma=1.2$ & $x_{1}(0)=[0.15,0]^{T}$ & $x_{2}(0)=[0.3,0]^{T}$ & $x_{3}(0)=[-0.15,0]^{T}$ \\
$x_{4}(0)=[-0.3,0]^{T}$ & & & & & \\
\hline
\end{tabular}

In Fig 2 (a), $y$ and $y_{d}$ are shown. It is obvious that the system has good tracking performance. In Fig 2 (b), the synchronization error signals are presented. The system entered the $5 \%$ error range after $0.55 \mathrm{~s}$, which indicates that the system met the preset goal of reaching a stable state within $0.8 \mathrm{~s}$ of the prescribed settling time. Fig 2 (c) and Fig 2 (d) denote system eventtriggered inputs and the inter-execution interval respectively. It implies that the Communication resources of MASs is economized effectively by reducing the update frequency of control signals.

\subsection{Practical Example}

Further, the following single-manipulator model is considered.

$J \ddot{q}_{i}+B \dot{q}_{i}+M g l \sin \left(q_{i}\right)=u$, where $J=1$ denotes the inertia. $M=0.1 \mathrm{~kg}$ is mass and $g=10 \mathrm{~kg} / N$ is acceleration of gravity. $l=1 \mathrm{~m}$ is length between center of mass and joint. And $B=1$ is coefficient of viscous friction. And the topology of practical example is as same as numerical example.

Similarly, Fig 3 (a) represents the trajectory of $y$ and $y_{d}$. In Fig 3 (b), the synchronization error signals were shown. Fig 3 (c) and Fig 3 (d) denote system eventtriggered inputs and the inter-execution interval. From Fig 3 (a) - Fig 3 (b), we know that the synchronization error of MASs is kept at a small level. In addition, the system entered an error range of $5 \%$ after $0.42 \mathrm{~s}$, indicating that the system had reached the preset goal of reaching a stable state within $0.8 s$ of the prescribed settling time. In addition, the saving effect of the communication resources of the considered system is better. 


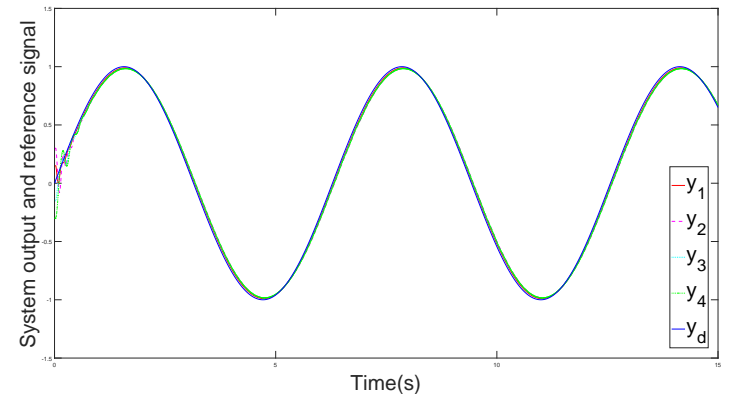

(a)

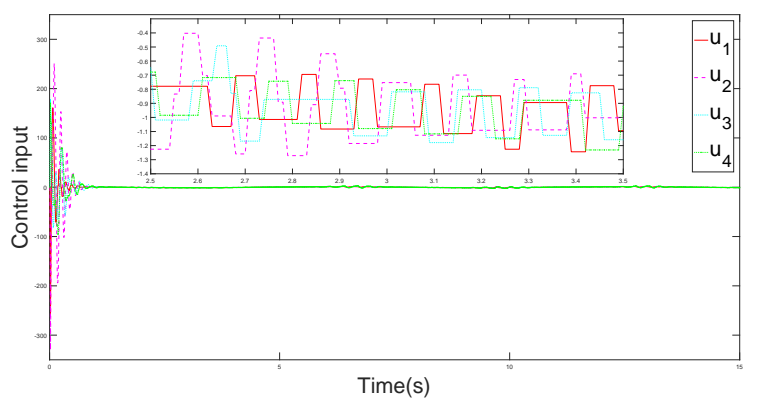

(c)

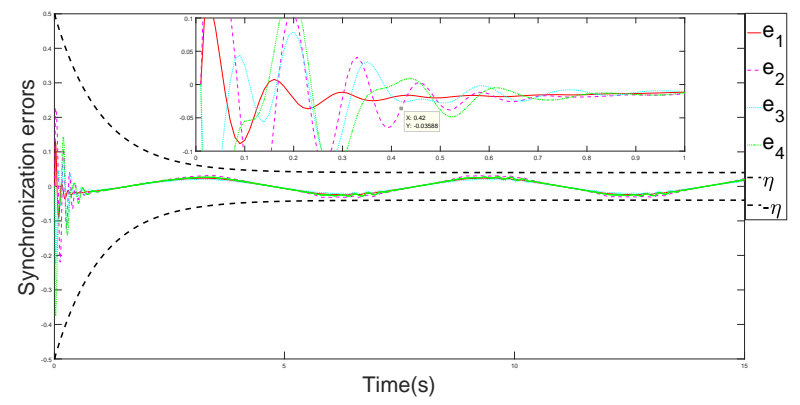

(b)
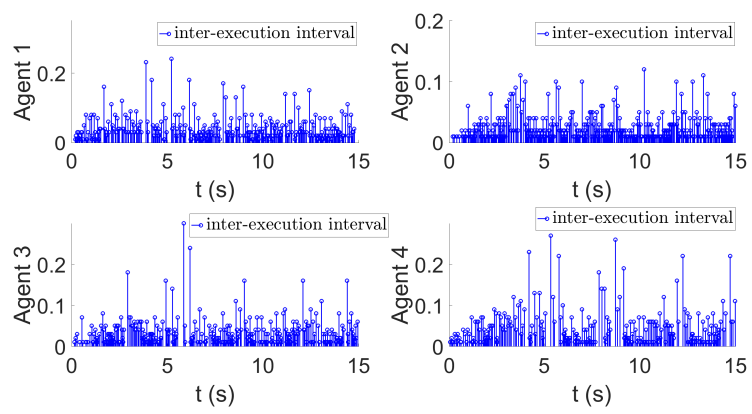

(d)

Fig. 3: Practical example: (a) $y$ and $y_{d}$; (b) Synchronization error signal; (c) System event-triggered inputs; (d) Inter-execution interval

\section{Conclusion}

In this research, the event-triggered prescribed setting time consensus control of uncertain nonlinear MASs with transient performance is investigated. By designing a performance function, the synchronization error converges to the predefined interval, which implies that the transient performance of MASs is guaranteed. Besides, with the aid of the designed nonlinear transformation function, the system can be stabilized within the prescribed setting time. Further, event-triggered strategy is introduced to economize the communication resources of the considered system. Simulation verification emphasizes its effectiveness.

Conflict of Interest: The authors declare that they have no conflict of interest.

\section{References}

1. Z. Zuo, Q. L. Han, B. Ning, et al, An overview of recent advances in fixed-time cooperative control of multiagent systems, IEEE Transactions on Industrial Informatics, 14(6) (2018) 2322-2334.
2. J. Qin, Q. Ma, Y Shi., et al, Recent advances in consensus of multi-agent systems: A brief survey, IEEE Transactions on Industrial Electronics, 64(6) (2016) 4972-4983.

3. F. Mehdifar, C. P. Bechlioulis, F. Hashemzadeh, et al, Prescribed performance distance-based formation control of Multi-Agent Systems, Automatica, 119 (2020) 109086.

4. W. Meng, P. X. Liu, Q. Yang, et al, Distributed Synchronization Control of Nonaffine Multiagent Systems with Guaranteed Performance, IEEE Transactions on Neural Networks and Learning Systems, 31(5) (2019) 1571-1580.

5. L. Wang, J. Dong, C. Xi, Event-triggered adaptive consensus for fuzzy output-constrained multi-agent systems with observers, Journal of the Franklin Institute, 357(1) (2020) 82-105.

6. C. Xi, J. Dong, Adaptive reliable guaranteed performance control of uncertain nonlinear systems by using exponent-dependent barrier Lyapunov function, International Journal of Robust and Nonlinear Control, 29(4) (2019) 1051-1062.

7. X. Meng, L. Xie, Y. C. Soh, Reset control for synchronization of multi-agent systems, Automatica, 104 (2019) 189-195.

8. M. Rehan, C. K. Ahn, M. Chadli, Consensus of one-sided Lipschitz multi-agents under input saturation, IEEE Transactions on Circuits and Systems II: Express Briefs, 67(4)(2019)745-749.

9. J. Wang, Z. Liu, Y. Zhang, et al, Adaptive neural control of a class of stochastic nonlinear uncertain systems with guaranteed transient performance, IEEE Transactions on Cybernetics, 50(7) (2019) 2971-2981.

10. W. Wang, D. Wang, Z. Peng, et al, Prescribed performance consensus of uncertain nonlinear strict-feedback 
systems with unknown control directions, IEEE Transactions on Systems, Man, and Cybernetics: Systems, 46(9) (2015) 1279-1286.

11. M. A. Razaq, M. Rehan, M. Tufail, et al, Multiple Lyapunov Functions Approach for Consensus of One-Sided Lipschitz Multi-Agents Over Switching Topologies and Input Saturation, IEEE Transactions on Circuits and Systems II: Express Briefs, 67(12)(2020)3267-3271.

12. K. Lu, Z. Liu, G. Lai, et al, Adaptive consensus tracking control of uncertain nonlinear multiagent systems with predefined accuracy, IEEE transactions on cybernetics, (2019) DOI: 10.1109/TCYB.2019.2933436.

13. L. Macellari, Y. Karayiannidis, D. V. Dimarogonas, Multi-agent second order average consensus with prescribed transient behavior, IEEE Transactions on Automatic Control, 62(10) (2016) 5282-5288.

14. E Rang. Isochrone families for second-order systems, IEEE Transactions on Automatic Control, 8(1) (1963) 64-65.

15. S. P. Bhat, D. S. Bernstein, Finite-time stability of homogeneous systems, Proceedings of the 1997 American Control Conference (Cat. No. 97CH36041). IEEE, 4(1997) 2513-2514.

16. S. P. Bhat, D. S. Bernstein, Lyapunov analysis of finitetime differential equations, Proceedings of 1995 American Control Conference-ACC'95. IEEE, 3(1995) 1831-1832.

17. S. Li, H. Du, X. Lin, Finite-time consensus algorithm for multi-agent systems with double-integrator dynamics, Automatica, 47(8) (2011) 1706-1712.

18. H. Wang, P. X. Liu, X. Zhao, et al, Adaptive fuzzy finitetime control of nonlinear systems with actuator faults, IEEE transactions on cybernetics, 50(5) (2019) 17861797.

19. S. Sui, C. L. P. Chen, S, Tong, Fuzzy adaptive finite-time control design for nontriangular stochastic nonlinear systems, IEEE Transactions on Fuzzy Systems, 27(1) (2018) 172-184.

20. H. Li, S. Zhao, W. He, et al, Adaptive finite-time tracking control of full state constrained nonlinear systems with dead-zone, Automatica, 100 (2019) 99-107.

21. X. Wang, S. Li, P. Shi, Distributed finite-time containment control for double-integrator multiagent systems, IEEE Transactions on Cybernetics, 44(9) (2013) 15181528.

22. F. Wang, B. Chen, Y. Sun, et al, Finite-time fuzzy control of stochastic nonlinear systems, IEEE transactions on cybernetics, 50(6) (2019) 2617-2626.

23. F. Lopez-Ramirez, A. Polyakov, D. Efimov, et al, Finitetime and fixed-time observer design: Implicit Lyapunov function approach, Automatica, 87(2018) 52-60.

24. Q. Lan, S. Li, J. Yang, Finite-time tracking control for a class of nonlinear systems with multiple mismatched disturbances, International Journal of Robust and Nonlinear Control, 30(10) (2020) 4095-4111.

25. B. Ning, Q. Han, Prescribed Finite-Time Consensus Tracking for Multiagent Systems with Nonholonomic Chained-Form Dynamics, IEEE Transactions on Automatic Control, 64(4) (2019) 1686-1693.

26. Y. Wang, Y. Song, D. J. Hill, et al, Prescribed-Time Consensus and Containment Control of Networked Multiagent Systems, IEEE Transactions on Cybernetics, 49(4) (2019) 1138-1147.

27. X. Ge, Q. L. Han, X. M. Zhang, et al, Distributed event-triggered estimation over sensor networks: A survey, IEEE Transactions on Cybernetics, 50(3) (2019) $1306-1320$
28. H. Liang, G. Liu, H. Zhang, et al, Neural-Network-Based Event-Triggered Adaptive Control of Nonaffine Nonlinear Multiagent Systems With Dynamic Uncertainties, IEEE Transactions on Neural Networks and Learning Systems, 2020.

29. L. Wu, Y. Gao, J. Liu, et al, Event-triggered sliding mode control of stochastic systems via output feedback, Automatica, 82 (2017) 79-92.

30. L. Xing, C. Wen, Z. Liu, et al, Event-triggered adaptive control for a class of uncertain nonlinear systems, IEEE transactions on automatic control, 62(4) (2016) 2071-2076.

31. L. Xing, C. Wen, Z. Liu, et al, Event-triggered output feedback control for a class of uncertain nonlinear systems, IEEE Transactions on Automatic Control, 64(1) (2018) 290-297.

32. E. Tian, Z. Wang, L. Zou, et al, Probabilistic-constrained filtering for a class of nonlinear systems with improved static event-triggered communication, International Journal of Robust and Nonlinear Control, 29(5) (2019) 1484-1498.

33. Y. Zhang, J. Sun, H. Liang, et al, Event-triggered adaptive tracking control for multiagent systems with unknown disturbances, IEEE transactions on cybernetics, 50(3) (2020) 890-901.

34. K. Liu, Z. Ji, X. Zhang, Periodic event-triggered consensus of multi-agent systems under directed topology, Neurocomputing, 385 (2020) 33-41.

35. W. Xu, D. W. C. Ho, J. Zhong, et al, Event/self-triggered control for leader-following consensus over unreliable network with DoS attacks, IEEE Transactions on Neural Networks and Learning Systems, 30(10) (2019) 31373149.

36. X. Wang, H. Su, Self-triggered leader-following consensus of multi-agent systems with input time delay, Neurocomputing, 330 (2019) 70-77.

37. X. Mi, Y. Zou, S. Li, et al, Self-triggered DMPC design for cooperative multiagent systems, IEEE Transactions on Industrial Electronics, 67(1) (2019) 512-520.

38. M. M. Polycarpous, P. A. Ioannou, A robust adaptive nonlinear control design, Automatica, 32(3) (1996) 423427.

39. B. Ren, P. P. San, S. S. Ge, et al, Adaptive dynamic surface control for a class of strict-feedback nonlinear systems with unknown backlash-like hysteresis, Conference on American Control Conference (2009) 4482-4487.

40. H. Zhang,F. L. Lewis, Adaptive cooperative tracking control of higher-order nonlinear systems with unknown dynamics, Automatica, 48(7) (2012) 1432-1439.

41. Y. Sun, B. Chen, C. Lin, et al, Adaptive neural control for a class of stochastic nonlinear systems by backstepping approach, Inf. Sci., 369(2016) 748-764. 
Figures

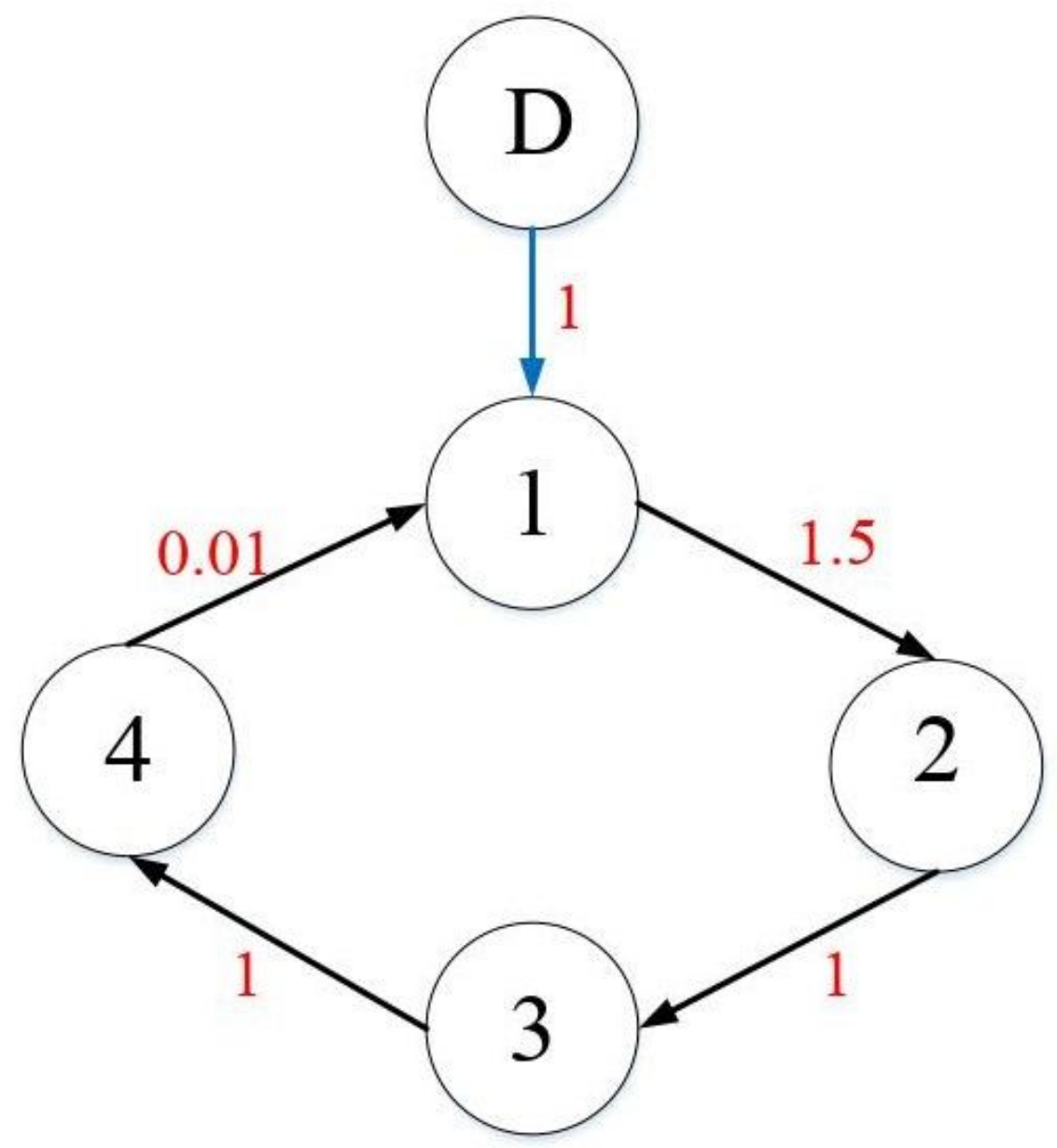

Figure 1

Topology of communication graph. 


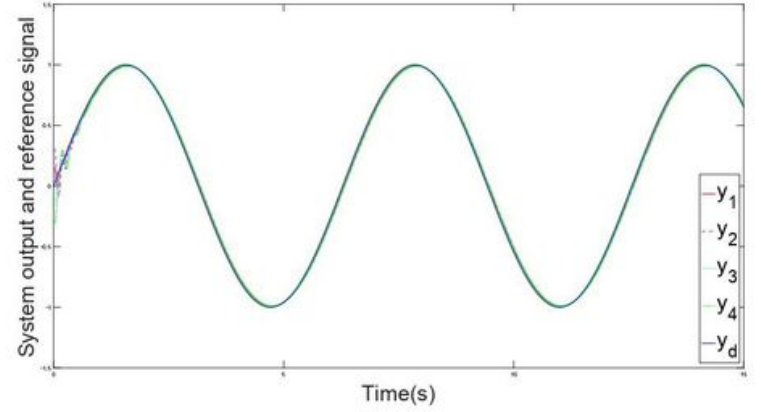

(a)

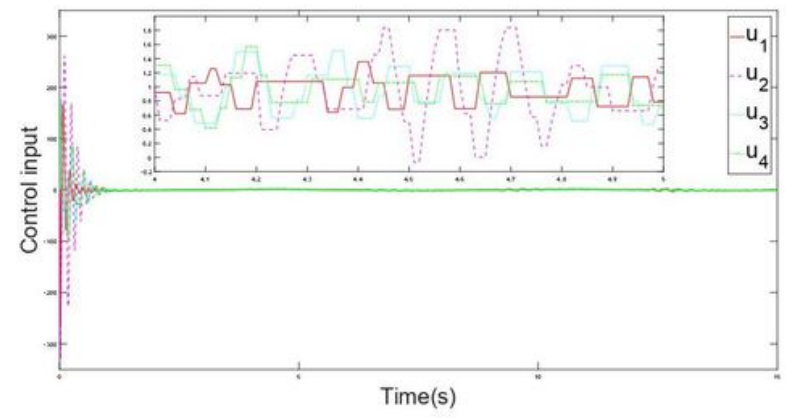

(c)

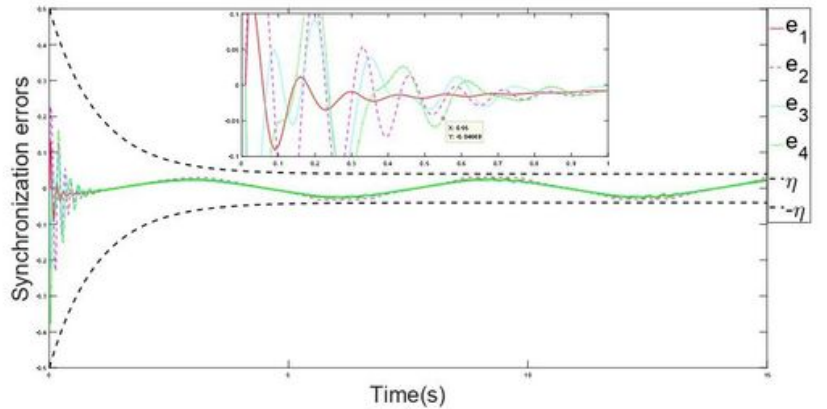

(b)
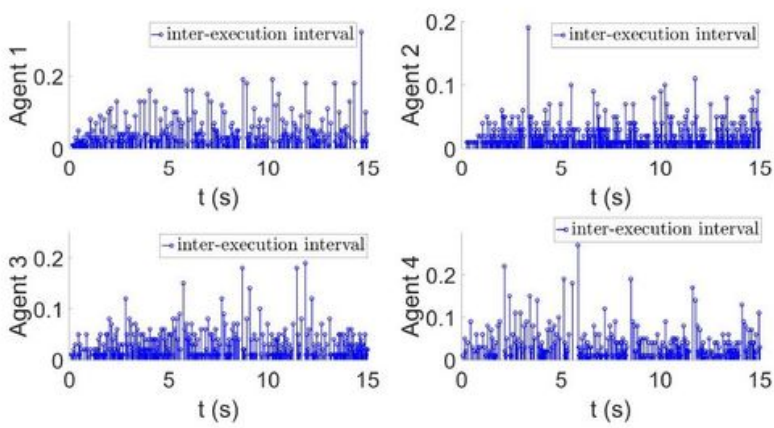

(d)

\section{Figure 2}

Numerical example: (a) y and yd; (b) Synchronization error signal; (c) System event-triggered inputs; (d) Inter-execution interval

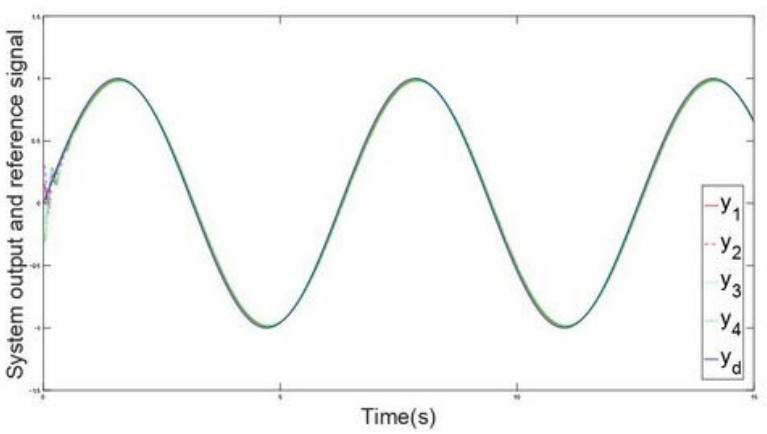

(a)

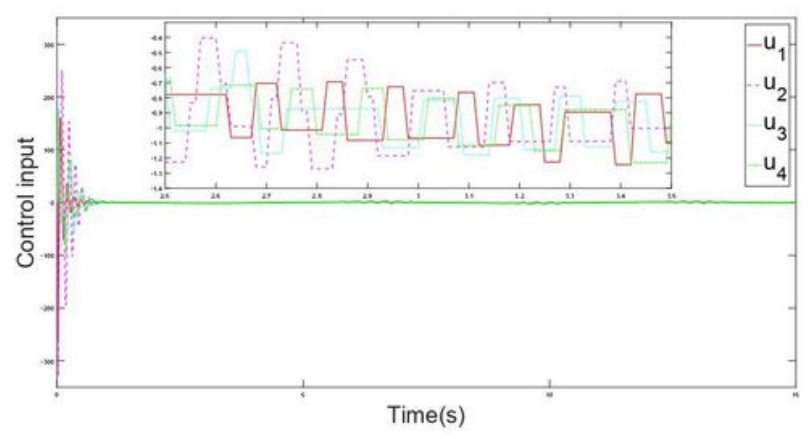

(c)

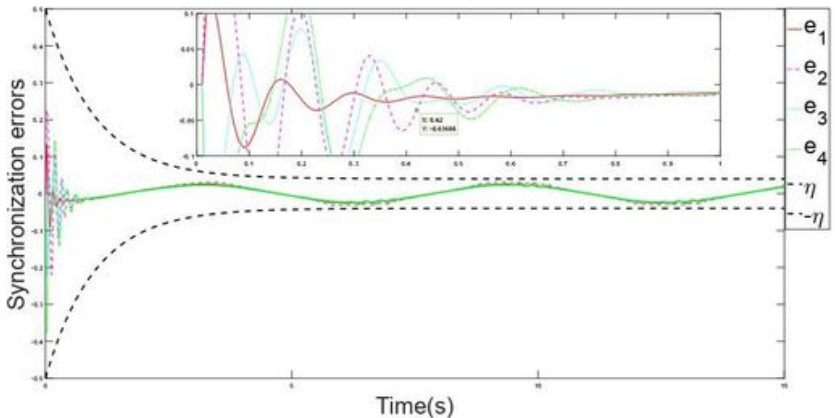

(b)
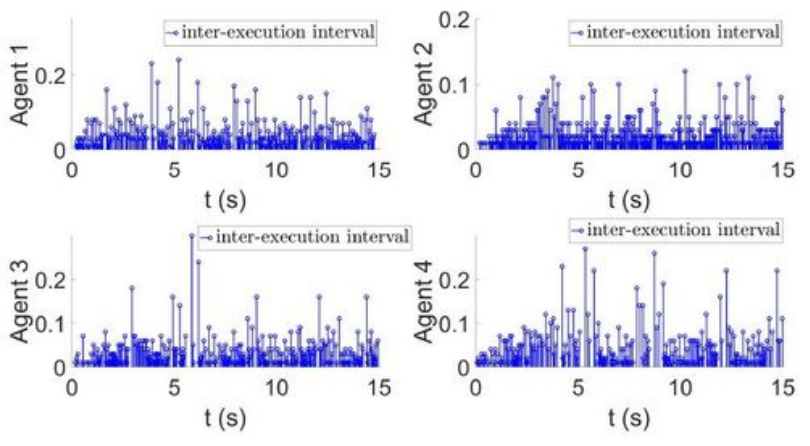

(d) 
Figure 3

Practical example: (a) y and yd; (b) Synchronization error signal; (c) System event-triggered inputs; (d) Inter-execution interval

\section{Supplementary Files}

This is a list of supplementary files associated with this preprint. Click to download.

- SourceFile.rar 